\title{
New Image Compression Algorithm using Haar Wavelet Transform
}

\author{
R. El Ayachi' ${ }^{1}$ B. Bouikhalene ${ }^{2}$, M. Fakir*1 \\ ${ }^{1}$ Department of Computer Science, Sultan Moulay Slimane University \\ ${ }^{2}$ Department of Mathematics\& Computer Science, Sultan Moulay Slimane University
}

\begin{abstract}
Article Info
Article history:

Received Jan 16, 2017

Revised Feb 27, 2017

Accepted Mar 20, 2017

ABSTRACT

The compression is a process of Image Processing which interested to change the information representation in order to reduce the stockage capacity and transmission time. In this work we propose a new image compression algorithm based on Haar wavelets by introducing a compression coefficient that controls the compression levels. This method reduces the complexity in obtaining the desired level of compression from the original image only and without using intermediate levels.
\end{abstract}

Keywords:

Compression

Haar wavelet

Image processing

Leve compression

Copyright $@ 2017$ Institute of Advanced Engineering and Science. All rights reserved.

\section{Corresponding Author:}

R. El Ayachi,

Departement of Computer Science,

Faculty of Science and Technology, Sultan Moulay Slimane University,

Email: rachid.elayachi@usms.ma

\section{INTRODUCTION}

The compression is an important domain in the scientific research, which was attracted the intention of the community scientific. The number of papers produced in this field proves this attraction. D. Salomon [1] treated in general the field of data compression. He presented the different methods and techniques concerning image compression, video compression and audio compression. E. Christophe \& al [2] focused on the proposal of a compression algorithm for hyperspectral images. This method is compared with the JPEG 2000 approach. Gaurav Vijayvargiya \& al [3] conducted an analysis of the various existing compression methods. This analysis focuses on two types of compression: lossless and loss. Sachin Dhawan [4] discussed the field of the image compression by presenting the principle, classes and different compression algorithms.

The compression is divided on two types: lossless and loss. The first type represent the possibility to restore identically the original image from the compressed image using the decompression processing. Therefore, the second type is an irreversible processing. There are many algorithms considering us loss compressing, like: Vector Quantization (VQ) [5], Discrete Cosine Transform (DCT) [6] and Haar wavelet $[7,8]$ which is the focus study of this paper.

Haar wavelet is an example of the compressing algorithm that produce the compressed image at a desired level. The goal of this study is to propose a new method based on the Haar wavelet algorithm in order to reduce the computational complexity.

The rest of the paper is organized as follows. Section 2 presents the principle of the Haar wavelet algorithm to calculate the compressed image at several levels. Section 3 describe a new image compression algorithm based on the Haar wavelets, this algorithm is controlled by a coefficient that produce the compressed image at a desired level by ignoring intermediate levels; these amendments allow the reduction 
of the computational complexity. Section 4 deab with the experimental results obtained. Finally, the conclusion is given in section 5 .

\section{HAAR WAVELET ALGORITHM}

Haar wavelet is a special function created by ALFREAD HAAR in 1909 [9], this function can be used in image processing, especially in compressing process [10].

The using of Haar wavelet in the compressing process allow obtaining the result at several levels (level 1, level 2, ... etc.). For each level, the result is divided into four parts (Figure 1): one representing the compressed image and the others containing the details (horizontal, vertical and diagonal).

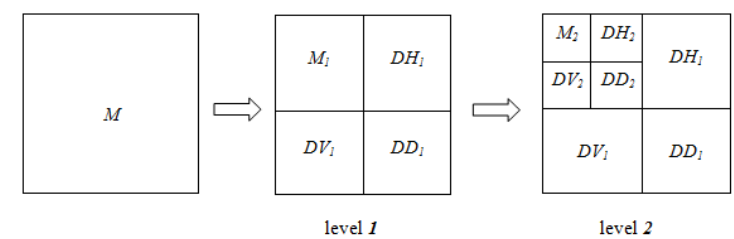

Figure 1. Operating of Haar wavelet compressing

Figure 1 represents the application of Haar wavelet to calculate the compressed image, where:

a. $M$ : Original image

b. $M_{l}$ : Compressed image at level 1

c. $D H_{l}, D V_{l}$ and $D D_{l}$ : Details at level 1

d. $M_{2}$ : Compressed image at level 2

e. $D \mathrm{H}_{2}, \mathrm{DV}_{2}$ and $\mathrm{DD}_{2}$ : Details at level 2

The computation of the compressed image at level $h$ is based on the compressed image obtained at level $h-1$. The details can be used in decompression process to restore the original image. formula:

The calculation of the compressed image at a level h using Haar wavelet, is based on the following

where

$$
S c_{h}=t\left(A_{h}\right) * M_{h-1} * A_{h}
$$

a. $h$ : Compression level

b. $S c_{h}$ : Compressed image at level $h$

c. $A_{h}$ : Haar wavelet coefficients at level $h$

d. $t\left(A_{h}\right)$ : Transposed of $A_{h}$

e. $M_{h-1}$ : Original image for level $h$

The Haar wavelet coefficients can be computed using the following formulas :

$$
\left\{\begin{array}{l}
A_{i j}=1 / 2 \text { if } j \leq \mathrm{N} / 2 \text { and } \mathrm{i}=2 * \mathrm{j}+1 \text { or } \mathrm{i}=2 * \mathrm{j} \\
A_{i j}=1 / 2 \text { if } \mathrm{N} / 2<j \leq \mathrm{N} \text { and } \mathrm{i}=2 *(\mathrm{j}-\mathrm{N} / 2)-1 \\
A_{i j}=-1 / 2 \text { if } \mathrm{N} / 2<j \leq \mathrm{N} \text { and } \mathrm{i}=2 *(\mathrm{j}-\mathrm{N} / 2) \\
A_{i j}=1 \text { if } \mathrm{i}>N \\
A_{i j}=0 \text { else }
\end{array}\right.
$$

where

a. $\quad N$ : Number of column

b. $i, j$ : Positive integers 


\section{PROPOSED COMPRESSION ALGORITHM}

The problem of the classical algorithm (section 2) is still in the dependence of the calculation of the compression at a level with the previous states (Figure 2), that is to say that to arrive at a level $\boldsymbol{h}$, we must started with a level $\boldsymbol{1}$, a level $2, \ldots$ until level $\boldsymbol{h}$.

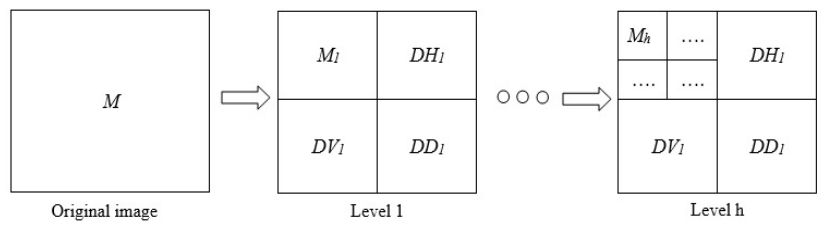

Figure 2. Classical computation of the compressed image at level $\mathrm{h}$

The question is how to compute the compressed image for a level $\boldsymbol{h}$ from an original image (Figure 3) without passing through intermediate levels? The solution is the purpose of the following.

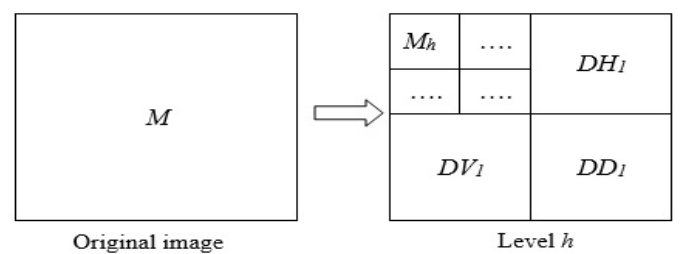

Figure 3. Proposed approach to compute the compressed image at level $\boldsymbol{h}$

To remedy this problem, we introduce a parameter $h$ in calculating the coefficients of the Haar wavelet as follows:

$$
\left\{\begin{array}{l}
A_{i j}=1 / 2^{h} \quad \text { if } j \leq \mathrm{N} / 2^{h} \text { and } \mathrm{i}=2^{h} * \mathrm{j}+1 \text { or } \mathrm{i}=2^{h} * \mathrm{j} \\
A_{i j}=1 / 2^{h} \text { if } \mathrm{N} / 2^{h}<j \leq \mathrm{N} \text { and } \mathrm{i}=2^{h} *\left(\mathrm{j}-\mathrm{N} / 2^{h}\right)-1 \\
A_{i j}=-1 / 2^{h} \text { if } \mathrm{N} / 2^{h}<j \leq \mathrm{N} \text { and } \mathrm{i}=2^{h} *\left(\mathrm{j}-\mathrm{N} / 2^{h}\right) \\
A_{i j}=1 \text { if } \mathrm{i}>N \\
A_{i j}=0 \text { else }
\end{array}\right.
$$

After this change, we use "Equation 1" to find the compressed image at level $h$, then, we multiply the result by a correction term according to the following formula:

$$
S c_{\text {res }}=S c_{h} * 2^{2(k-1)}
$$

where

a. $S c_{h}$ : is the compressed image at level $\boldsymbol{h}$ before correction

b. $S c_{r e s}:$ is the compressed image at level $\boldsymbol{h}$ After correction

\section{EXPERIMENTAL RESULTS AND DISCUSSION}

Comparative study of a set of algorithms to choose the best, based on the criterion of complexity. The latter means the amount of resources needed for processing an input using an algorithm. The main resources are measured time (number of instructions used) and space (amount of storage space required). In this paper, we use the first resource.

In this section, we present the results of compression of a set of images using the principle Haar wavelets. Different compressions will be calculated using algorithms mentioned in sections 2 and 3 . 
Figure 4, Figure 5, Figure 6 and Figure 7 represents the results obtained using the classical application of Haar wavelet algorithm at three levels (Level 1, Level 2 and Level 3).
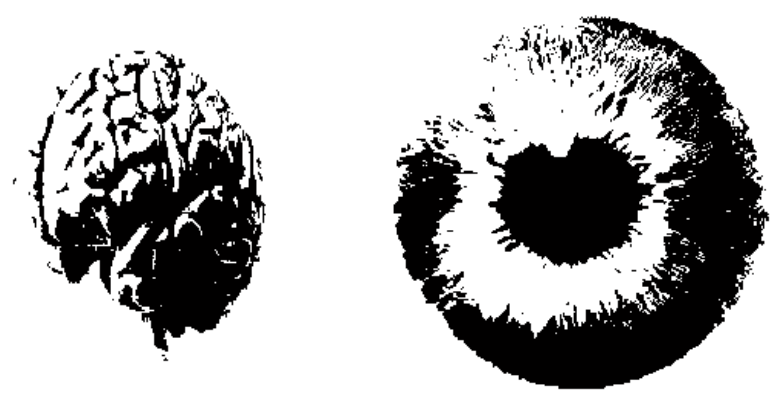

Figure 4. Original images

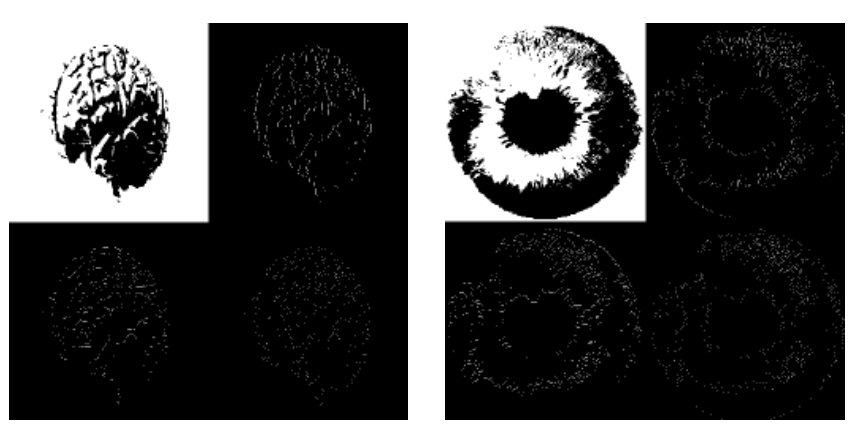

Figure 5. Compression at level 1 using classical algorithm
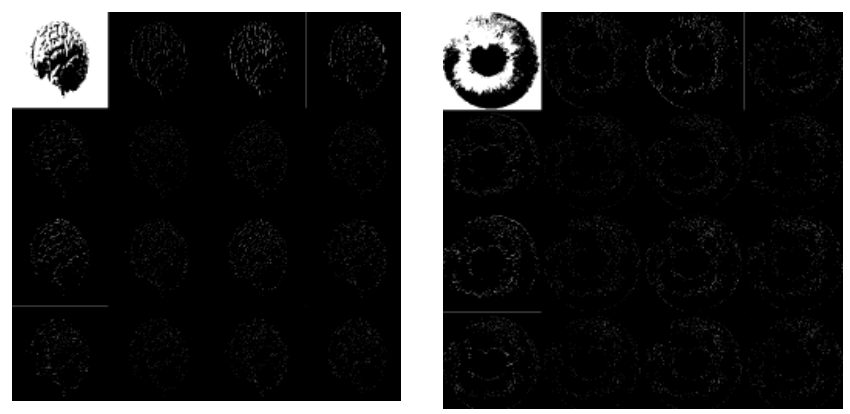

Figure 6. Compression at level 2 using classical algorithm
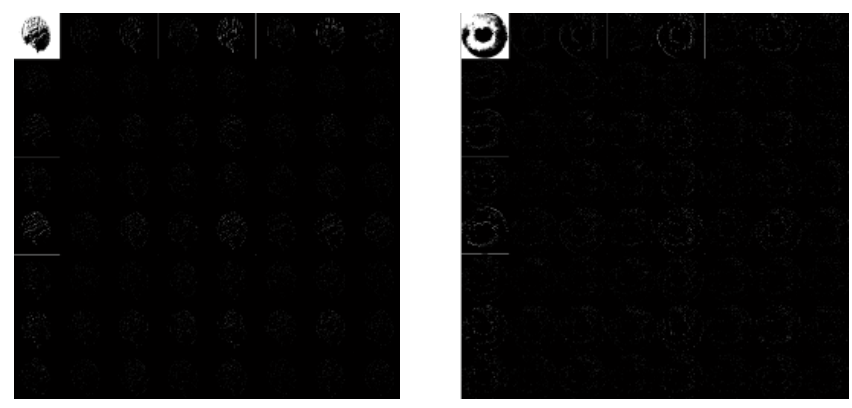

Figure 7. Compression at level 3 using classical algorithm 
Figure 8, Figure 9 and Figure 10 represents the results obtained using the proposed algorithm of Haar wavelet at three levels (Level 1, Level 2 and Level 3).

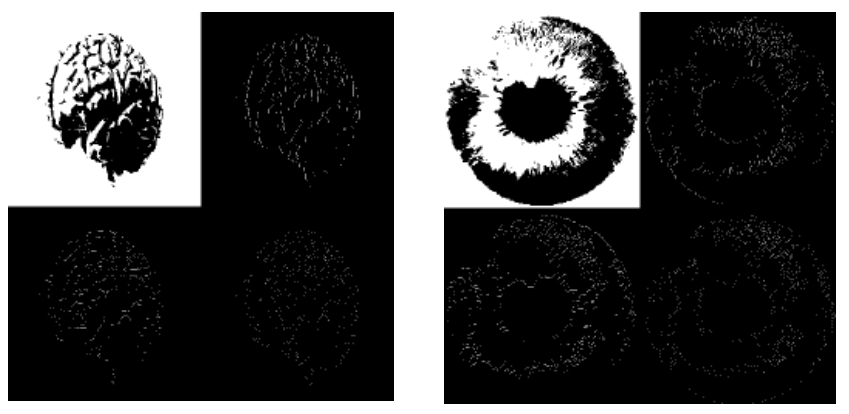

Figure 8. Compression at level 1 using proposed algorithm
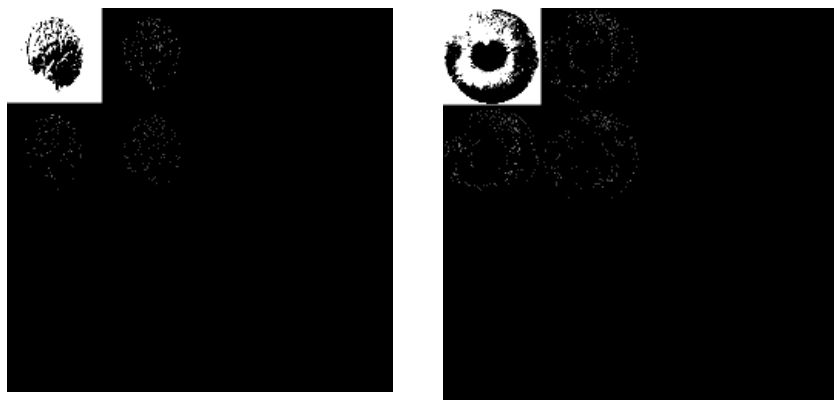

Figure 9. Compression at level 2 using proposed algorithm
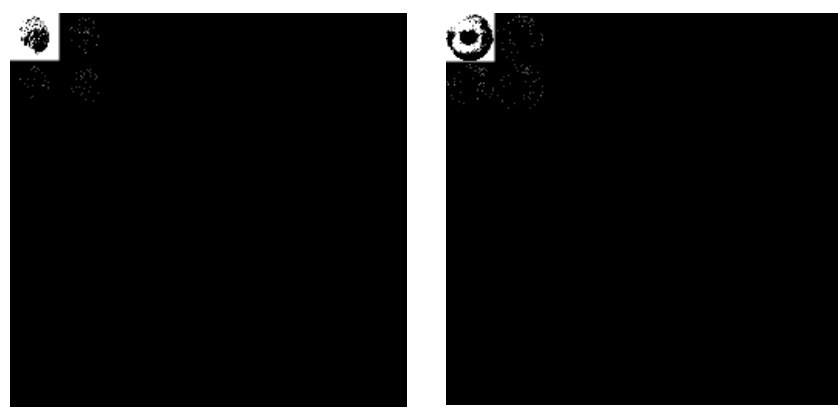

Figure 10. Compression at level 3 using proposed algorithm

After noticing the similarity of compression results for both algorithms (classical and proposed), we calculate the complexity in order to determine the influence of improvements made.

Table 1 shows the execution time required to compute the compressed image at three levels (Level 1, Level 2 and Level 3) for both algorithms.

Table 1. Execution time

\begin{tabular}{lccc}
\hline $\begin{array}{c}\text { Haar wavelet } \\
\text { algorithm }\end{array}$ & Level 1 & $\begin{array}{c}\text { Execution time (s) } \\
\text { Level 2 }\end{array}$ & Level 3 \\
\hline Classical & 0.4101 & 0.5867 & 0.8675 \\
Improvement & 0.3860 & 0.4122 & 0.5556 \\
\hline
\end{tabular}


From Table 1, it is clear that the performance of the improved algorithm time is less strictly to the execution time of the classical algorithm for the three levels.

Table 2 represents the number of instructions using to calculate the compressed image at three levels (Level 1, Level 2 and Level 3) for both algorithms. For this case, the number of instructions concerning calculation of the Haar wavelet coefficients is not counted.

Table 2. Number of instructions

\begin{tabular}{cccc}
\hline Haar wavelet & \multicolumn{3}{c}{ Number of instructions } \\
algorithm & Level 1 & Level 2 & Level 3 \\
\hline Classical & $2 \times(\mathrm{N} 3 \times(\mathrm{N}-1))$ & $(2 \times(\mathrm{N} 3 \times(\mathrm{N}-1))) \times 2$ & $(2 \times(\mathrm{N} 3 \times(\mathrm{N}-1))) \times 3$ \\
Improvement & $2 \times(\mathrm{N} 3 \times(\mathrm{N}-1))$ & $2 \times(\mathrm{N} 3 \times(\mathrm{N}-1))$ & $2 \times(\mathrm{N} 3 \times(\mathrm{N}-1))$ \\
\hline
\end{tabular}

\section{CONCLUSION}

Image compression becomes a crucial field of image treatment. In this paper, we proposed a new compression approach based on the Haar wavelets and controlled by a coefficient. This new method allows to compute the compressed image at desired level from the original image only. In this case, the computational complexity are reduced because the intermediate levels are ignored in the compression process.

\section{REFERENCES}

[1] D. Salomon, "Data Compression: The Complete Reference," Springer. 2004.

[2] E. Christophe; C. Thiebaut; W. A. Pearlman; C. Latry; D. Lebedeff , "Zerotree-based Compression Algorithm for Spaceborne Hyperspectral Sensor,” On-Board Payload Data Compression Workshop - OBPDC. 2008.

[3] Gaurav Vijayvargiya; Sanjay Silakari; Rajeev Pandey , "A Survey: Various Techniques of Image Compression," International Journal of Computer Science and Information Security (IJCSIS), vol.11, no.10, 2013.

[4] Sachin Dhawan, A Review of Image Compression and Comparison of its Algorithms. IJECT. 2011; 2(1).

[5] S.Sathappan. "A Vector Quantization Technique for Image Compression using Modified Fuzzy Possibilistic CMeans with Weighted Mahalanobis Distance," International Journal of Innovative Research in Computer and Communication Engineering, vol.1, no.1, 2013.

[6] A.M.Raid; W.M.Khedr; M. A. El-dosuky; Wesam Ahmed, "Jpeg Image Compression Using Discrete Cosine Transform - A Survey. International Journal of Computer Science \& Engineering Survey (IJCSES), vol.5, no.2, 2014.

[7] Piotr Porwik; Agnieszka Lisowska , "The Haar-Wavelet Transform in Digital Image Processing: Its Status and Achievements," Machine Graphics \& Vision, vol.13, no.1/2, pp.79-98, 2004.

[8] Myung-Sin Song, "Wavelet Image Compression, Mathematics Subject Classification,” Primary 42C40. 1991.

[9] Ronald A. DeVore; Björn Jawerth; Bradley J. Lucier , "Image Compression Through Wavelet Transform Coding," IEEE Transactions On Information Theory, vol.38, no.2, 1992.

[10] Kamrul Hasan Talukder; Koichi Harada , "Haar Wavelet Based Approach for Image Compression and Quality Assessment of Compressed Image," IAENG International Journal of Applied Mathematics , vol.36, no.1, 2007.

[11] Arikatla Hazarathaiah; B Prabhakara Rao , "Medical Image Compression using Lifting based New Wavelet Transforms," International Journal of Electrical and Computer Engineering (IJECE) , vol.4, no.5, pp.741-750, October 2014. 